\title{
Risk Factors of postoperative shivering after oral-maxillofacial surgery
}

\author{
Masanori Tsukamoto, Takashi Hitosugi, Takeshi Yokoyama
}

\author{
Department of Dental Anesthesiology, Kyushu University Hospital, Fukuoka, Japan \\ Department of Dental Anesthesiology, Faculty of Dental Science, Kyushu University, Fukuoka, Japan
}

\begin{abstract}
$\langle$ Background 〉
Postoperative shivering is involuntary movement during the early recovery following general anesthesia, which is frequent complication of anesthesia. It occurs with core temperature around $36.0^{\circ} \mathrm{C}$ of the patients recovering from general anesthesia. We had often experienced postoperative shivering in patients after osteotomy of maxilla and/or mandible. Furthermore, it occurred even in normothermic patients during perioperative period. However, there are few reports about postoperative shivering after these surgery.

In this study, we investigated the risk factors of postoperative shivering in oral-maxillofacial surgery retrospectively.
\end{abstract}

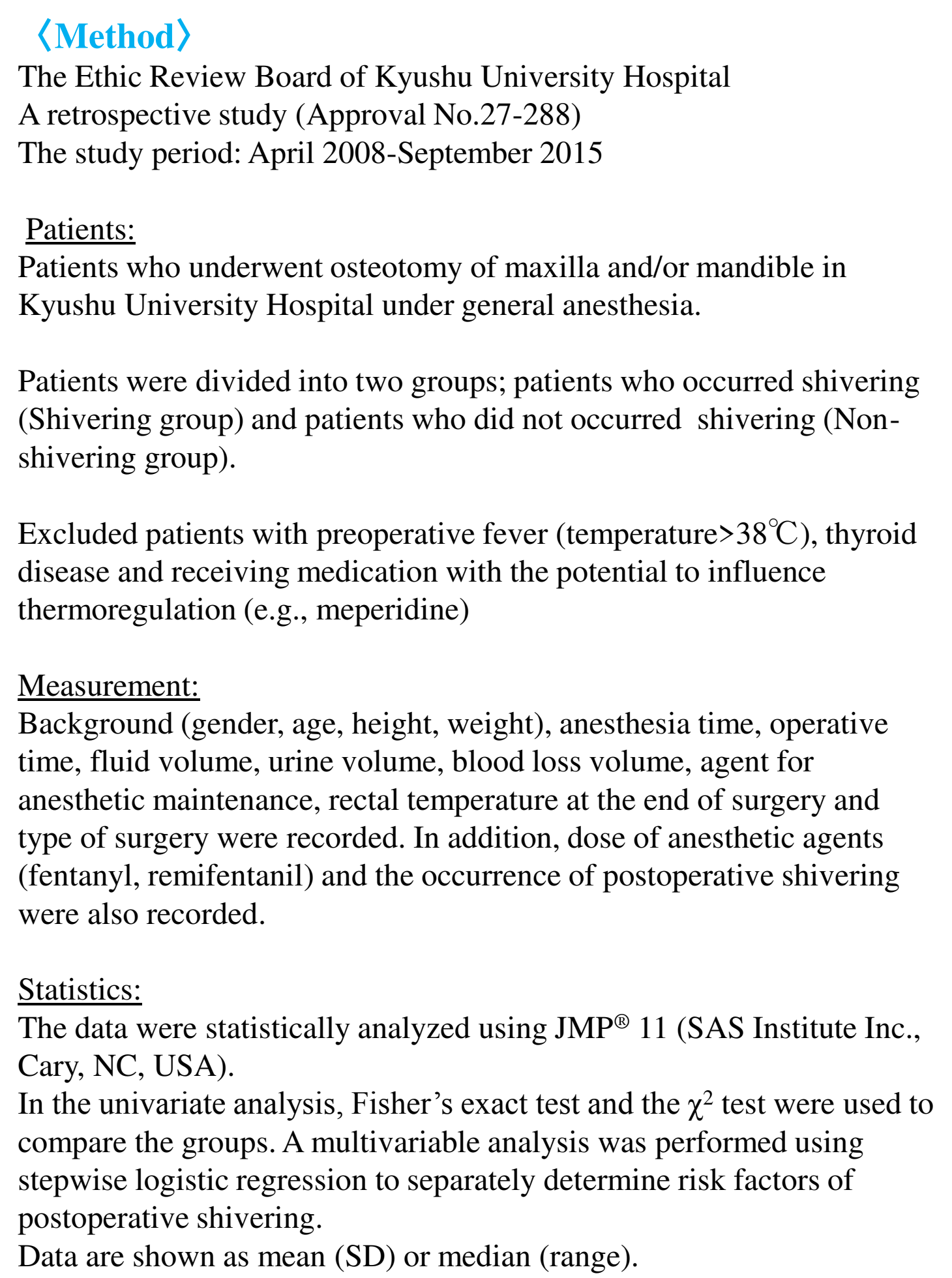

The Ethic Review Board of Kyushu University Hospital

A retrospective study (Approval No.27-288)

The study period: April 2008-September 2015

\section{Patients:}

Patients who underwent osteotomy of maxilla and/or mandible in Kyushu University Hospital under general anesthesia.

Patients were divided into two groups; patients who occurred shivering (Shivering group) and patients who did not occurred shivering (Nonshivering group).

Excluded patients with preoperative fever (temperature $>38^{\circ} \mathrm{C}$ ), thyroid disease and receiving medication with the potential to influence thermoregulation (e.g., meperidine)

The data were statistically analyzed using JMP ${ }^{\circledR} 11$ (SAS Institute Inc., Cary, NC, USA).

In the univariate analysis, Fisher's exact test and the $\chi^{2}$ test were used to compare the groups. A multivariable analysis was performed using stepwise logistic regression to separately determine risk factors of postoperative shivering.

Data are shown as mean (SD) or median (range).
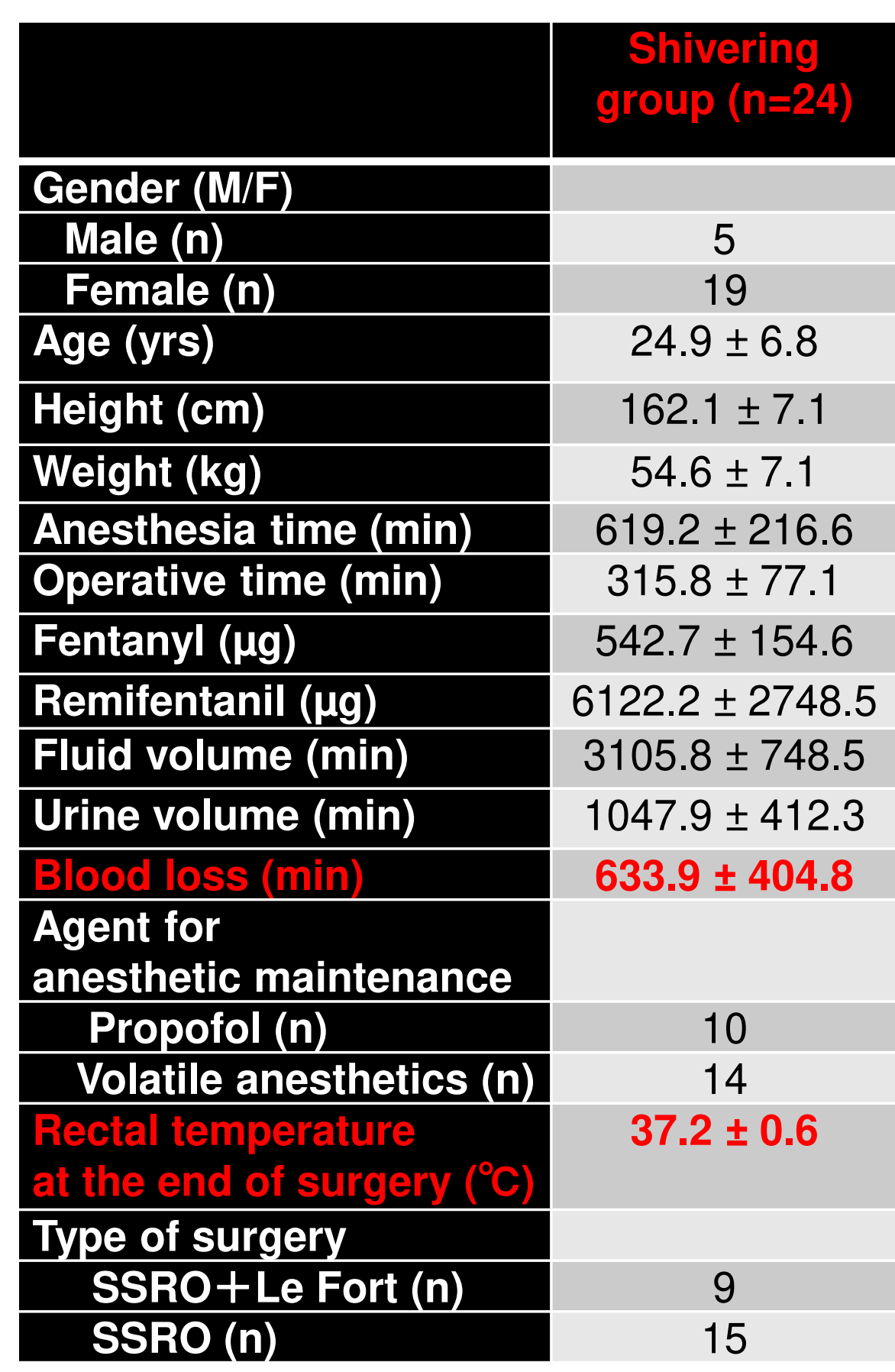

Non-shivering group $(\mathrm{n}=209)$

$24.9 \pm 6.8$ 69 140 $162.1 \pm 7.1$ $26.6 \pm 9.1$

$54.6 \pm 7.1$ $163.5 \pm 7.7$ $619.2 \pm 216.6$ $56.1 \pm 9.4$ 0.53 $315.8 \pm 77.1$ $371.8 \pm 231.8$ $263.2 \pm 90.0$ $542.7 \pm 154.6$ $6122.2 \pm 2748.5$ $3105.8 \pm 748.5$ $429.3 \pm 229.7$ 0.53 $1047.9 \pm 412.3$ $633.9 \pm 404.8$ $4463.4 \pm 3380.4$ $2633.7 \pm 2049.3$ $747.3 \pm 571.1$ 0.90 0.83 0.30 0.28 0.56 0.74 0.76 0.75 0.24 $<0.01$ 0.14 $367.0 \pm 312.6$

\begin{tabular}{|c|c|c|}
\hline 10 & 47 & \\
\hline $37.2 \pm 0.6$ & 162 & \\
\hline & $37.5 \pm 0.5$ & $<0.01$ \\
\hline 15 & & 0.88 \\
\hline
\end{tabular}

Fig.1 Univariate analysis: the risk factors of postoperative shivering

\begin{tabular}{|l|c|c|c|}
\hline & Odds ratio & $\mathbf{9 5 \%} \mathbf{~ C l}$ & p value \\
\hline Blood loss (min) & 0.997733 & $0.996546-0.99886$ & $<0.01$ \\
\hline $\begin{array}{l}\text { Rectal temperature at the end of } \\
\text { surgery }\left({ }^{\circ} \mathrm{C}\right)\end{array}$ & $\mathbf{2 . 5 6 0 2 7 7}$ & $1.236774-5.327362$ & 0.01 \\
\hline
\end{tabular}

Fig.2 Multivariate analysis (Logistic regression): the risk factors of postoperative shivering

\section{〈Results and Discussion〉}

Two hundred thirty three cases were suitable for this study. Twenty four of 233 cases (11.5\%) had postoperative shivering. Based on the univariate statistical analysis for all recorded data, we determined that 17 factors were potentially related to the occurrence of postoperative shivering (Fig.1).

Among the 17 potentially relevant factors that were included in the stepwise logistic regression analysis, 15 were removed because they proved to be no significant predictors at the $5 \%$ level.

The occurrence of postoperative shivering was correlated with blood loss (Shivering group: $633.9 \pm 404.8 \mathrm{ml}$ vs Non-shivering group: $367.0 \pm 312.6 \mathrm{ml}$ ) ( $\mathrm{p}<0.01$ ) and Rectal temperature at the end of surgery (Shivering group: $37.2 \pm 0.6^{\circ} \mathrm{C}$ vs Non-shivering group: $37.5 \pm 0.5^{\circ} \mathrm{C}$ ) ( $<<0.01$ ). Two variables were associated with postoperative shivering. Rectal temperature at the end of surgery was the highest risk factor (Fig.2).

Rectal temperature at the end of surgery with 4 patients was more than $38.0^{\circ} \mathrm{C}$. The second was blood loss.

Our study demonstrated that mean rectal temperature at the end of surgery was $37.2 \pm 0.6^{\circ} \mathrm{C}$ in patients with postoperative shivering, including 4 patients with rectal temperature more than $38.0^{\circ} \mathrm{C}$.

Postoperative shivering might occur when a discrepancy of the set point between normal thresholds and postoperative thresholds at the emergence from general anesthesia.

\section{〈Conclusion〉}

We should pay attention to the postoperative shivering not only in patients with hypothermia but also in patients with much bleeding. 